\title{
CHIKUNGUNYA AND SYSTEMIC LUPUS ERYTHEMATOSUS - A CASE SERIES
}

Rebeca Xavier Linhares Silva ${ }^{1}$, Lia Lima de Azevedo ${ }^{1}$, Evânia Claudino Queiroga de Figueiredo 1, ${ }^{1}$, Marcus Ivanovith Fernandes ${ }^{1}$, Bárbara Letícia de Moura Pinheiro ${ }^{1}$, Thamiris Miranda Granja ${ }^{1}$, Antônio da Silva Ramos Segundo Neto ${ }^{1}$, Priscylla Emylly Lacerda de Sousa ${ }^{1}$

1.Universidade Federal de Campina Grande, Campina Grande (PB), Brazil.

*Corresponding author: evaniaclaudinofig@gmail.com

\section{BACKGROUND}

Chikungunya ( $\mathrm{CHIK}$ ) is a disease that affects thousands of people annually and causes debilitating joint pain. It is transmitted by mosquitoes from the genus Aedes, mainly by Aedes aegypti and Aedes albopictus. In the last 15 years, there have been an increase in the frequency of chikungunya surges that can be related to a greater incidence of more severe forms of the disease than previously described, besides having occurred atypical forms, in which patients expressed, among other autoimmune diseases, the systemic lupus erythematosus (SLE). The SLE is a complex autoimmune disease, with varied clinical presentations and multifactorial etiology, in which genetic and environmental factors interact determining the susceptibility to the disease. As environmental factors, there are viral infections that can intensify undesirable immune responses, stimulating specific immune cells.

\section{METHODS}

This study identified patients with previous CHIK diagnosis that developed SLE in a university hospital in the state of Paraíba, Brazil, through a cross-sectional retrospective study in medical records of the Alcides Carneiro University Hospital, selecting a case series of patients who manifested SLE after CHIK from 2014 to 2020, which were conducted inpatient or outpatient. The medical records were evaluated based on a questionnaire with specific criteria based on SLICC 2012 e ACR/EULAR 2019 for the diagnosis of SLE, and on clinical, epidemiological and serological criteria for the diagnosis of $\mathrm{CHIK}$.

\section{RESULTS}

Six cases were selected for analysis. There was a predominance of $4 / 6$ females (66.7\%) with a mean age of SLE diagnosis of 34.8 years. The mean time interval between chikungunya infection and the development of SLE was 1.8 year. There were $\lg \mathrm{G}$ and/or IgM reagents for chikungunya in 4/6 (66.7\%); in 2/6 (33.3\%) the diagnosis was made based on clinical and epidemiological criteria. Joint involvement was present in 6/6 (100\%) of the patients with CHIK and progressed to synovitis in 5/6 (83.3\%) when SLE was diagnosed. Every patient studied presented ANA and the anti-dsDNA reagents, this being the profile of autoantibodies highlighted. Non-scarring alopecia has occurred in $4 / 6$ (66.7\%) patients. Renal involvement occurred in $2 / 6$ (33.3\%), both with biopsy for class IV lupus nephritis.

\section{CONCLUSION}

The relevance of this study is reinforced as it is a topic with incipient and scarce literature, for supporting the continuity of research and alerting health teams to the identification of autoimmune diseases related to chikungunya, with a focus on SLE.

\section{KEYWORDS}

Chikungunya, Systemic lupus erythematosus, Autoimmunity. 